International Journal of Pure and Applied Mathematics

Volume 108 No. 4 2016, 751-766

ISSN: 1311-8080 (printed version); ISSN: 1314-3395 (on-line version)

url: http://www.ijpam.eu

doi: 10.12732/ijpam.v108i4.2

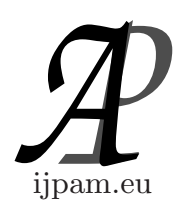

\title{
COMMON COUPLED FIXED POINTS FOR FOUR MAPS USING $\alpha$-ADMISSIBLE FUNCTIONS IN COMPLEX VALUED $b$ - METRIC SPACES
}

\author{
K.P.R. Rao ${ }^{1}$, V.C.C. Raju ${ }^{2}$, P. Ranga Swamy ${ }^{3}$, Sk. Sadik ${ }^{4}$ \\ ${ }^{1}$ Department of Mathematics \\ Acharya Nagarjuna University \\ Nagarjuna Nagar, 522 510, A.P., INDIA \\ ${ }^{2}$ Department of Mathematics \\ University of Botswana \\ Private Bag, UB 00704, Gaborone, BOTSWANA \\ ${ }^{3}$ Department of Mathematics \\ St. Ann's College of Engineering and Technology, \\ Chirala, 523 187, Prakasam (Dt.), A.P., INDIA \\ ${ }^{4}$ Department of Mathematics \\ Sir CRR College of Engineering, Eluru \\ West Godhawari, 534007, Andhra Pradesh, INDIA
}

\begin{abstract}
In this paper, we obtain a unique common coupled fixed point theorem for four self maps using $\alpha$-admissible function in complex valued $b$-metric spaces. Also we give an example to illustrate our main theorem.
\end{abstract}

AMS Subject Classification: 54H25, 47H10

Key Words: complete metric spaces, $\alpha$-admissible functions, compatible mappings

\section{Introduction and Preliminaries}

It is a well-known fact that the mathematical results regarding fixed points of contraction type mappings are very useful for determining the existence and

Received: November 25, 2015

Published: August 16, 2016

${ }^{\S}$ Correspondence author
(C) 2016 Academic Publications, Ltd.

url: www.acadpubl.eu 
uniqeness of solutions to various mathematical models.

Azam et al. [1] introduced the notion of complex valued metric space which is a generalization of the classical metric space and obtained sufficent conditions for the existence of common fixed points of a pair of mappings satisfying a rational contractive condition.Later several authors proved fixed and common fixed point theorems in complex valued metric spaces, for example, refer [3, 4, $6,8,11,12,14,16,20,21,28]$. Recently some authors,for example[7, 13, 17, 22, $24,25,26]$, obtained coupled and common coupled fixed point point theorems for a pair of mappings in complex valued metric spaces.

In this paper,we prove a unique common fixed point theorem for two pairs of mappings satisfying a contractive condition of rational type in the frame work of complex valued $b$-metric spaces using $\alpha$-admissible function. The proved result generalizes and extends some of the results of [13, 22, 24, 25].

To begin with, we recall some basic definitions, notations and results.

Throughout this paper $\mathcal{R}, \mathcal{R}^{+}, \mathcal{N}$ and $\mathcal{C}$ denote the set of all real numbers, non-negative real numbers, positive integers and complex numbers respectively.First we refer the following preliminaries.

Let $z_{1}, z_{2} \in \mathcal{C}$. Define a partial order $\precsim$ on $\mathcal{C}$ follows:

$z_{1} \precsim z_{2}$ if and only if $\operatorname{Re}\left(z_{1}\right) \leq \operatorname{Re}\left(z_{2}\right), \operatorname{Im}\left(z_{1}\right) \leq \operatorname{Im}\left(z_{2}\right)$.

Thus $z_{1} \precsim z_{2}$ if one of the following holds:

(1) $\operatorname{Re}\left(z_{1}\right)=\operatorname{Re}\left(z_{2}\right)$ and $\operatorname{Im}\left(z_{1}\right)=\operatorname{Im}\left(z_{2}\right)$,

$(2) \operatorname{Re}\left(z_{1}\right)<\operatorname{Re}\left(z_{2}\right)$ and $\operatorname{Im}\left(z_{1}\right)=\operatorname{Im}\left(z_{2}\right)$,

(3) $\operatorname{Re}\left(z_{1}\right)=\operatorname{Re}\left(z_{2}\right)$ and $\operatorname{Im}\left(z_{1}\right)<\operatorname{Im}\left(z_{2}\right)$,

(4) $\operatorname{Re}\left(z_{1}\right)<\operatorname{Re}\left(z_{2}\right)$ and $\operatorname{Im}\left(z_{1}\right)<\operatorname{Im}\left(z_{2}\right)$.

Clearly $z_{1} \precsim z_{2} \Rightarrow\left|z_{1}\right| \leq\left|z_{2}\right|$.

Definition 1. ([1]) Let $X$ be a non empty set. A function $d: X \times X \rightarrow \mathcal{C}$ is called a complex valued metric on $X$ if for all $x, y, z \in X$ the following conditions are satisfied:

(i) $0 \precsim d(x, y)$ and $d(x, y)=0$ if and only if $x=y$;

(ii) $d(x, y)=d(y, x)$;

(iii) $d(x, y) \precsim d(x, z)+d(z, y)$.

The pair $(X, d)$ is called a complex valued metric space.

Now, we briefly review the notation about complex valued b-metric spaces. 
Definition 2. ([9]) Let $X$ be a non empty set and $s \geq 1$. A function $d: X \times X \rightarrow \mathbb{C}$ is called a complex valued $b$ - metric on $X$ if for all $x, y, z \in X$ the following conditions are satisfied:

(i) $0 \precsim d(x, y)$ and $d(x, y)=0$ if and only if $x=y$;

(ii) $d(x, y)=d(y, x)$;

(iii) $d(x, y) \precsim s[d(x, z)+d(z, y)]$.

The pair $(X, d)$ is called a complex valued $b$ - metric space.

Remark 1. Let $(X, d)$ be a complex valued $b$-metric space. Then

(i) $|d(x, y)|$ or $|d(u, v)|<|1+d(x, y)+d(u, v)|, \forall x, y, u, v \in X$.

(ii) If $x \neq y$ then $|d(x, y)|>0$.

(iii) For $0 \leq k<1$ and $z, w \in \mathcal{C}$, if $|z| \leq k|w|$ and $|w| \leq k|z|$ then $z=w=0$.

Definition 3. ([9]) Let $(X, d)$ be a complex valued $b$-metric space.

(i) A point $x \in X$ is called interior point of a set $A \subseteq X$ whenever there exists $0 \prec r \in \mathcal{C}$ such that $B(x, r)=\{y \in X: d(x, y) \prec r\} \subseteq A$.

(ii) A point $x \in X$ is called a limit point of a set $A \subseteq X$ whenever there exists $0 \prec r \in \mathcal{C}$ such that $B(x, r) \cap(X-A) \neq \phi$.

(iii) A subset $B \subseteq X$ is called open whenever each point of $B$ is an interior point of $B$.

(iv) A subset $B \subseteq X$ is called closed whenever each limit point of $B$ is in $B$.

(v) The family $F=\{B(x, r): x \in X$ and $0 \prec r\}$ is a sub basis for a topology on $X$. We denote this complex topology by $\tau_{c}$. Indeed, the topology $\tau_{c}$ is Hausdorff.

Let $\left\{x_{n}\right\}$ be a sequence in $X$ and $x \in X$.If for every $c \in \mathcal{C}$ with $0 \preceq c$ there is $n_{0} \in \mathcal{N}$ such that for all $n>n_{0}, d\left(x_{n}, x\right) \prec c$, then $\left\{x_{n}\right\}$ is said to be convergent to $x$ and $x$ is the limit point of $\left\{x_{n}\right\}$. We denote this by $\lim _{n \rightarrow \infty} x_{n}=x$ or $x_{n} \rightarrow x$ as $n \rightarrow \infty$. If for every $c \in \mathcal{C}$ with $0 \prec c$ there is $n_{0} \in \mathcal{N}$ such that for all $n>n_{0}, d\left(x_{n}, x_{n+m}\right) \prec c$, where $m \in \mathcal{N}$, then $\left\{x_{n}\right\}$ is called a Cauchy sequence in $(X, d)$. If every Cauchy sequence is convergent in $(X, d)$ then $(X, d)$ is called a complete complex valued $b$-metric space. We require the follwing lemmas. 
Lemma 1.([9]) Let $(X, d)$ be a complex valued $b$-metric space and let $\left\{x_{n}\right\}$ be a sequence in X.Then $\left\{x_{n}\right\}$ converges to $\mathrm{x}$ if and only if $\left|d\left(x_{n}, x\right)\right| \rightarrow 0$ as $n \rightarrow \infty$.

Lemma 2.([9]) Let $(X, d)$ be a complex valued $b$-metric space and let $\left\{x_{n}\right\}$ be a sequence in X.Then $\left\{x_{n}\right\}$ is a Cauchy sequence if and only if

$\left|d\left(x_{n}, x_{n+m}\right)\right| \rightarrow 0$ as $n, m \rightarrow \infty$.

One can easily prove the following lemma

Lemma 3.Let $(X, d)$ be a complex valued $b$-metric space and let $\left\{x_{n}\right\}$ and $\left\{y_{n}\right\}$ be sequences in $X$ converging to $x$ and $y$ respectively. Then

(i) $\frac{1}{s}|d(x, z)| \leq \lim _{n \rightarrow \infty}\left|d\left(x_{n}, z\right)\right| \leq s|d(x, z)|$ for all $z \in X$,

(ii) $\frac{1}{s^{2}}|d(x, y)| \leq \lim _{n \rightarrow \infty}\left|d\left(x_{n}, y_{n}\right)\right| \leq s^{2}|d(x, y)|$.

Recently Bhaskar and Lakshmikantham [23] introduced the concept of coupled fixed point and discussed some problems of the uniqueness of a coupled fixed point and applied their results to the problems of the existence and uniqueness of a solution for the periodic boundary value problems. Later Lakshmikantham and Ciric [27] proved some coupled coincidence and coupled common fixed point results in partially ordered metric spaces.

Definition 4. Let $X$ be a non empty set and $F: X \times X \rightarrow X$ and $S: X \rightarrow X$.

(i)([27]) An element $(x, y) \in X \times X$ is called a common coupled fixed point of mappings $F$ and $S$ if $x=S x=F(x, y)$ and $y=S y=F(y, x)$.

(ii)([10]) The pair $(F, S)$ is called $w$-compatible if $S(F(x, y))=F(S x, S y)$ and $S(F(y, x))=F(S y, S x)$, whenever $S x=F(x, y)$ and $S y=F(y, x)$.

Now we extend the definition of compatible maps introduced by Jungck [5] in metric spaces to complex valued $b$-metric spaces to the maps $F: X \times X \rightarrow X$ and $S: X \rightarrow X$ as follows.

Definition 5. Let $(X, d)$ be a complex valued $b$-metric space and $F$ : $X \times X \rightarrow X$ and $S: X \rightarrow X$. Then the pair $(F, f)$ is said to be compatible if

(i) $\lim _{n \rightarrow \infty}\left|d\left(S\left(F\left(x_{n}, y_{n}\right)\right), F\left(S x_{n}, S y_{n}\right)\right)\right|=0$ and

(ii) $\lim _{n \rightarrow \infty}\left|d\left(S\left(F\left(y_{n}, x_{n}\right)\right), F\left(S y_{n}, S x_{n}\right)\right)\right|=0$

whenever there exist sequences $\left\{x_{n}\right\}$ and $\left\{y_{n}\right\}$ in $X$ such that $F\left(x_{n}, y_{n}\right) \rightarrow$ $t, S x_{n} \rightarrow t$ and $F\left(y_{n}, x_{n}\right) \rightarrow t^{\prime}, S y_{n} \rightarrow t^{\prime}$ for some $t, t^{\prime} \in X$.

Samet et al. [2] introduced the notion of $\alpha$-admissible mappings as follows 
Definition 6. ([2]) Let $X$ be a non empty set, $T: X \rightarrow X$ and $\alpha$ : $X \times X \rightarrow \mathcal{R}^{+}$be mappings. Then $T$ is called $\alpha$-admissible if for all $x, y \in X$, we have $\alpha(x, y) \geq 1$ implies $\alpha(T x, T y) \geq 1$.

In the sequel,Kaushik et al. [18]introduced the following $\alpha$-admissible mapping concept which is a generalization of the concept introduced by Mursaleen et al. [15].

Definition 7. ([18])Let $X$ be a nonempty set and $\alpha: X^{2} \times X^{2} \rightarrow \mathcal{R}^{+}$be a function.Let $F: X \times X \rightarrow X$ and $S: X \rightarrow X$ be mappings. Then $F$ and $S$ are said to be $\alpha$-admissible if

$$
\alpha((S x, S y),(S u, S v)) \geq 1 \Rightarrow \alpha((F(x, y), F(y, x)),(F(u, v), F(v, u))) \geq 1
$$

for all $x, y, u, v \in X$.

If $S=I$ (Identity map), then the above definition is the concept of Mursaleen et al. [15].

In this paper, we give an extension of the above definition for four maps.

Definition 8. Let $X$ be a nonempty set and $\alpha: X^{2} \times X^{2} \rightarrow \mathcal{R}^{+}$. Let $F, G: X \times X \rightarrow X$ and $S, T: X \rightarrow X$ be mappings. Then we say that the pair $(S, T)$ is $\alpha$-admissible with respect to the pair $(F, G)$ if

(i) $\alpha((S x, S y),(T u, T v)) \geq 1 \Rightarrow \alpha((F(x, y), F(y, x)),(G(u, v), G(v, u))) \geq$ 1 ,

(ii) $\alpha((T x, T y),(S u, S v)) \geq 1 \Rightarrow \alpha((G(x, y), G(y, x)),(F(u, v), F(v, u))) \geq$ 1 , for all $x, y, u, v \in X$.

Combining all the above notions,now we prove our main result.

\section{Main Result}

Theorem 9. Let $(X, d)$ be a complete complex valued $b$-metric space with $s \geq 1$.Let $\alpha: X^{2} \times X^{2} \rightarrow \mathcal{R}^{+}$be a function and $F, G: X \times X \rightarrow X$ and $S, T: X \rightarrow X$ be mappings satisfying the following:

(9.1) $F(X \times X) \subseteq T(X), G(X \times X) \subseteq S(X)$,

$$
\begin{gathered}
\alpha((S x, S y),(T u, T v)) s^{4} d(F(x, y), G(u, v)) \precsim a_{1} d(S x, T u) \\
+a_{2} d(S y, T v)+a_{3} d(S x, F(x, y))
\end{gathered}
$$




$$
\begin{aligned}
& +a_{4} d(S y, F(y, x))+a_{5} d(T u, G(u, v))+a_{6} d(T v, G(v, u)) \\
& +a_{7} \frac{d(S x, F(x, y)) d(T u, G(u, v))}{1+s^{4} d(S x, T u)+s^{4} d(S y, T v)} \\
& +a_{8} \frac{d(T u, F(x, y)) d(S x, G(u, v))}{1+s^{4} d(S x, T u)+s^{4} d(S y, T v)}
\end{aligned}
$$

for all $x, y, u, v \in X$, where $a_{i}, i=1,2, \ldots ., 8$ are non-negative real numbers such that $\sum_{i=1}^{8} a_{i}<\frac{1}{s}$

(9.3) (a) $\alpha\left(\left(S x_{1}, S y_{1}\right),\left(F\left(x_{1}, y_{1}\right), F\left(y_{1}, x_{1}\right)\right)\right) \geq 1$,

(b) $\alpha\left(\left(S y_{1}, S x_{1}\right),\left(F\left(y_{1}, x_{1}\right), F\left(x_{1}, y_{1}\right)\right)\right) \geq 1$,

(c) $\alpha\left(\left(F\left(x_{1}, y_{1}\right), F\left(y_{1}, x_{1}\right)\right),\left(S x_{1}, S y_{1}\right)\right) \geq 1$ and

(d) $\alpha\left(\left(F\left(y_{1}, x_{1}\right), F\left(x_{1}, y_{1}\right)\right),\left(S y_{1}, S x_{1}\right)\right) \geq 1$ for some $x_{1}, y_{1} \in X$,

(9.4) the pair $(S, T)$ is $\alpha$-admissible with respect to the pair $(F, G)$,

$(9.5)(a)$ the pair $(F, S)$ is compatible, $S$ is continuous and the pair $(G, T)$ is wcompatible and if there exist sequences $\left\{z_{n}\right\},\left\{w_{n}\right\}$ in $X$ such that

$$
\begin{aligned}
& \alpha\left(\left(z_{n}, w_{n}\right),\left(z_{n+1}, w_{n+1}\right)\right) \geq 1, \\
& \alpha\left(\left(z_{n+1}, w_{n+1}\right),\left(z_{n}, w_{n}\right)\right) \geq 1, \\
& \alpha\left(\left(w_{n}, z_{n}\right),\left(w_{n+1}, z_{n+1}\right)\right) \geq 1, \\
& \alpha\left(\left(w_{n+1}, z_{n+1}\right),\left(w_{n}, z_{n}\right)\right) \geq 1,
\end{aligned}
$$

with $z_{n} \rightarrow z$ and $w_{n} \rightarrow w$ imply 1 ,

(i) $\alpha\left(\left(S z_{2 n}, S w_{2 n}\right),\left(z_{2 n+1}, w_{2 n+1}\right)\right) \geq 1, \alpha\left(\left(S w_{2 n}, S z_{2 n}\right),\left(w_{2 n+1}, z_{2 n+1}\right)\right) \geq$

(ii) $\alpha\left((z, w),\left(z_{2 n+1}, w_{2 n+1}\right)\right) \geq 1, \alpha\left((w, z),\left(w_{2 n+1}, z_{2 n+1}\right)\right) \geq 1$,

(iii) $\alpha(z, w),(z, w)) \geq 1, \alpha(w, z),(w, z)) \geq 1$,

(iv) $\alpha((z, w),(T z, T w)) \geq 1, \alpha((w, z),(T w, T z)) \geq 1$ for all $n \in \mathcal{N}$.

(or)

(9.5)(b) the pair $(G, T)$ is compatible,T is continuous and the pair $(F, S)$ is wcompatible and if there exist sequences $\left\{z_{n}\right\},\left\{w_{n}\right\}$ in $X$ such that

$$
\begin{aligned}
& \alpha\left(\left(z_{n}, w_{n}\right),\left(z_{n+1}, w_{n+1}\right)\right) \geq 1, \\
& \alpha\left(\left(z_{n+1}, w_{n+1}\right),\left(z_{n}, w_{n}\right)\right) \geq 1, \\
& \alpha\left(\left(w_{n}, z_{n}\right),\left(w_{n+1}, z_{n+1}\right)\right) \geq 1,
\end{aligned}
$$




$$
\alpha\left(\left(w_{n+1}, z_{n+1}\right),\left(w_{n}, z_{n}\right)\right) \geq 1
$$

with $z_{n} \rightarrow z$ and $w_{n} \rightarrow w$ imply 1 ,

(i) $\alpha\left(\left(z_{2 n}, w_{2 n}\right),\left(T z_{2 n+1}, T w_{2 n+1}\right)\right) \geq 1, \alpha\left(\left(w_{2 n}, z_{2 n}\right),\left(T w_{2 n+1}, T z_{2 n+1}\right)\right) \geq$

(ii) $\left.\left.\alpha\left(z_{2 n}, w_{2 n}\right),(z, w)\right) \geq 1, \alpha\left(w_{2 n}, z_{2 n}\right),(w, z)\right) \geq 1$,

(iii) $\alpha((z, w),(z, w)) \geq 1, \alpha((w, z),(w, z)) \geq 1$,

(iv) $\alpha((S z, S w),(z, w)) \geq 1, \alpha((S w, S z),(w, z)) \geq 1$ for all $n \in \mathcal{N}$.

Then $F, G, S$ and $T$ have a common coupled fixed point.

(9.6) Further if we assume that $\alpha\left((z, w),\left(z^{\prime}, w^{\prime}\right)\right) \geq 1$ and

$\alpha\left((w, z),\left(w^{\prime}, z^{\prime}\right)\right) \geq 1$ whenever $(z, w)$ and $\left(z^{\prime}, w^{\prime}\right)$ are common coupled fixed points of $F, G, S$ and $T$ then $F, G, S$ and $T$ have a unique common coupled fixed point in $X \times X$.

Proof. Let $x_{1}$ and $y_{1}$ be in $X$ satisfying (9.3). Now define the sequences $\left\{z_{n}\right\}$ and $\left\{w_{n}\right\}$ from (9.1) as follows:

$$
\begin{aligned}
z_{2 n+1} & =F\left(x_{2 n+1}, y_{2 n+1}\right)=T x_{2 n+2}, \\
z_{2 n+2} & =G\left(x_{2 n+2}, y_{2 n+2}\right)=S x_{2 n+3}, \\
w_{2 n+1} & =F\left(y_{2 n+1}, x_{2 n+1}\right)=T y_{2 n+2}, \\
w_{2 n+2} & =G\left(y_{2 n+2}, x_{2 n+2}\right)=S y_{2 n+3}, \text { for } n=0,1,2,3, \cdots
\end{aligned}
$$

From $(9.3)(a)$, we have

$$
\begin{aligned}
& \alpha\left(\left(S x_{1}, S y_{1}\right),\left(F\left(x_{1}, y_{1}\right), F\left(y_{1}, x_{1}\right)\right)\right) \geq 1 \\
& \Rightarrow \alpha\left(\left(S x_{1}, S y_{1}\right),\left(T x_{2}, T y_{2}\right)\right) \geq 1, \text { from definition of }\left\{z_{n}\right\} \text { and }\left\{w_{n}\right\} \\
& \Rightarrow \alpha\left(\left(F\left(x_{1}, y_{1}\right), F\left(y_{1}, x_{1}\right)\right),\left(G\left(x_{2}, y_{2}\right), G\left(y_{2}, x_{2}\right)\right)\right) \geq 1, \text { from }(9.4) \\
& \Rightarrow \alpha\left(\left(z_{1}, w_{1}\right),\left(z_{2}, w_{2}\right)\right) \geq 1 \text { from definition of }\left\{z_{n}\right\} \text { and }\left\{w_{n}\right\} \\
& \Rightarrow \alpha\left(\left(T x_{2}, T y_{2}\right),\left(S x_{3}, S y_{3}\right)\right) \geq 1, \text { from definition of }\left\{z_{n}\right\} \text { and }\left\{w_{n}\right\} \\
& \Rightarrow \alpha\left(\left(G\left(x_{2}, y_{2}\right), G\left(y_{2}, x_{2}\right)\right),\left(F\left(x_{3}, y_{3}\right), F\left(y_{3}, x_{3}\right)\right)\right) \geq 1, \text { from }(9.4) \\
& \Rightarrow \alpha\left(\left(z_{2}, w_{2}\right),\left(z_{3}, w_{3}\right)\right) \geq 1 .
\end{aligned}
$$

Continuing in this way, we have

$$
\alpha\left(\left(z_{n}, w_{n}\right),\left(z_{n+1}, w_{n+1}\right)\right) \geq 1, \quad \forall n .
$$

Similarly from $(9.3)(c),(9.3)(b)$ and $(9.3)(d)$ we can obtain

$$
\alpha\left(\left(z_{n+1}, w_{n+1}\right),\left(z_{n}, w_{n}\right)\right) \geq 1 .
$$




$$
\begin{aligned}
& \alpha\left(\left(w_{n}, z_{n}\right),\left(w_{n+1}, z_{n+1}\right)\right) \geq 1 . \\
& \alpha\left(\left(w_{n+1}, z_{n+1}\right),\left(w_{n}, z_{n}\right)\right) \geq 1 .
\end{aligned}
$$

Let $R_{n}=\max \left\{d\left(z_{n}, z_{n+1}\right), d\left(w_{n}, w_{n+1}\right)\right\}$.

Case (i) Suppose $R_{2 m}=0$ for some $m$. Then $z_{2 m}=z_{2 m+1}$ and $w_{2 m}=$ $w_{2 m+1}$. Now

$$
\begin{aligned}
\alpha\left(\left(S x_{2 m+1}, S y_{2 m+1}\right),\right. & \left.\left(T x_{2 m+2}, T y_{2 m+2}\right)\right)=\alpha\left(\left(z_{2 m}, w_{2 m}\right),\left(z_{2 m+1}, w_{2 m+1}\right)\right) \geq 1, \\
& \text { from }(1), \\
d\left(z_{2 m+1}, z_{2 m+2}\right)= & d\left(F\left(x_{2 m+1}, y_{2 m+1}\right), G\left(x_{2 m+2}, y_{2 m+2}\right)\right) \\
\precsim & \alpha\left(\left(S x_{2 m+1}, S y_{2 m+1}\right),\left(T x_{2 m+2}, T y_{2 m+2}\right)\right) \\
& s^{4} d\left(F\left(x_{2 m+1}, y_{2 m+1}\right), G\left(x_{2 m+2}, y_{2 m+2}\right)\right) \\
\precsim & a_{1} d\left(z_{2 m}, z_{2 m+1}\right)+a_{2} d\left(w_{2 m}, w_{2 m+1}\right)+a_{3} d\left(z_{2 m}, z_{2 m+1}\right) \\
& +a_{4} d\left(w_{2 m}, w_{2 m+1}\right)+a_{5} d\left(z_{2 m+1}, z_{2 m+2}\right) \\
& +a_{6} d\left(w_{2 m+1}, w_{2 m+2}\right) \\
& +a_{7} \frac{d\left(z_{2 m}, z_{2 m+1}\right) d\left(z_{2 m+1}, z_{2 m+2}\right)}{1+s^{4} d\left(z_{2 m}, z_{2 m+1}\right)+s^{4} d\left(w_{2 m}, w_{2 m+1}\right)} \\
& +a_{8} \frac{d\left(z_{2 m+1}, z_{2 m+1}\right) d\left(z_{2 m}, z_{2 m+2}\right)}{1+s^{4} d\left(z_{2 m}, z_{2 m+1}\right)+s^{4} d\left(w_{2 m}, w_{2 m+1}\right)} \\
= & a_{5} d\left(z_{2 m+1}, z_{2 m+2}\right)+a_{6} d\left(w_{2 m+1}, w_{2 m+2}\right) .
\end{aligned}
$$

Thus $\left|d\left(z_{2 m+1}, z_{2 m+2}\right)\right| \leq\left(a_{5}+a_{6}\right)\left|R_{2 m+1}\right|$.

Similarly using (3), we have $\left|d\left(w_{2 m+1}, w_{2 m+2}\right)\right| \leq\left(a_{5}+a_{6}\right)\left|R_{2 m+1}\right|$.

Thus $\left|R_{2 m+1}\right| \leq\left(a_{5}+a_{6}\right)\left|R_{2 m+1}\right|$ which in turn yields that $z_{2 m+1}=z_{2 m+2}$ and $w_{2 m+1}=w_{2 m+2}$.

Continuing in this way, we get $z_{2 m}=z_{2 m+1}=z_{2 m+2}=\cdots$ and $w_{2 m}=w_{2 m+1}=w_{2 m+2}=\cdots$.

Hence $\left\{z_{n}\right\}$ and $\left\{w_{n}\right\}$ are Cauchy sequences in $(X, d)$.

Case (ii): Assume that $R_{n} \neq 0$ for all $n$.

As in Case (i), using Remark 1(i), we have

$$
\mid d\left(z_{2 n+1}, z_{2 n+2}\left|\leq\left(a_{1}+a_{2}+a_{3}+a_{4}\right)\right| R_{2 n}\left|+\left(a_{5}+a_{6}+a_{7}\right)\right| R_{2 n+1} \mid\right.
$$

and

$$
\mid d\left(w_{2 n+1}, w_{2 n+2}\left|\leq\left(a_{1}+a_{2}+a_{3}+a_{4}\right)\right| R_{2 n}\left|+\left(a_{5}+a_{6}+a_{7}\right)\right| R_{2 n+1} \mid .\right.
$$


Thus $\left|R_{2 n+1}\right| \leq k\left|R_{2 n}\right|$,where $k_{1}=\frac{a_{1}+a_{2}+a_{3}+a_{4}}{1-a_{5}-a_{6}-a_{7}}$.

Similarly using (2) and (4), we have $\left|R_{2 n+2}\right| \leq k_{2}\left|R_{2 n+1}\right|$, where $k_{2}=$ $\frac{a_{1}+a_{2}+a_{5}+a_{6}}{1-a_{3}-a_{4}-a_{7}}$. Let $k=\max \left\{k_{1}, k_{2}\right\}$. Thus

$$
\begin{aligned}
\left|R_{n}\right| & \leq k\left|R_{n-1}\right|, \quad n=2,3, \ldots \\
& \leq k^{n-1}\left|R_{1}\right|
\end{aligned}
$$

For $m>n$,using (5) we have

$$
\begin{aligned}
\left|d\left(z_{n}, z_{m}\right)\right| & \leq s\left|d\left(z_{n}, z_{n+1}\right)\right|+s^{2}\left|d\left(z_{n+1}, z_{n+2}\right)\right|+\ldots \ldots+s^{m-n-1}\left|d\left(z_{m-1}, z_{m}\right)\right| \\
& \leq\left[s k^{n-1}+s^{2} k^{n}+\ldots \ldots+s^{m-n-1} k^{m-2}\right]\left|R_{1}\right| \\
& \leq\left[(s k)^{n-1}+(s k)^{n}+\ldots \ldots+(s k)^{m-2}\right]\left|R_{1}\right| \\
& \leq \frac{(s k)^{n-1}}{1-s k}\left|R_{1}\right| \rightarrow 0 \text { as } n, m \rightarrow \infty, \text { since } s k<1 .
\end{aligned}
$$

Thus $\left\{z_{n}\right\}$ is a Cauchy sequence in $(X, d)$. Similarly we can show that $\left\{w_{n}\right\}$ is a Cauchy sequence in $(X, d)$. Since $(X, d)$ is complete,there exist $z, w \in X$ such that $\lim _{n \rightarrow \infty}\left|d\left(z_{n}, z\right)\right|=0$ and $\lim _{n \rightarrow \infty}\left|d\left(w_{n}, w\right)\right|=0$ from Lemma 1. Thus

$$
\begin{aligned}
z=\lim _{n \rightarrow \infty} F\left(x_{2 n+1}, y_{2 n+1}\right)=\lim _{n \rightarrow \infty} G\left(x_{2 n+2},\right. & \left.y_{2 n+2}\right) \\
& =\lim _{n \rightarrow \infty} T x_{2 n}=\lim _{n \rightarrow \infty} S x_{2 n+1}
\end{aligned}
$$

and

$$
\begin{aligned}
w=\lim _{n \rightarrow \infty} F\left(y_{2 n+1}, x_{2 n+1}\right)=\lim _{n \rightarrow \infty} G\left(y_{2 n+2},\right. & \left.x_{2 n+2}\right) \\
& =\lim _{n \rightarrow \infty} T y_{2 n}=\lim _{n \rightarrow \infty} S y_{2 n+1}
\end{aligned}
$$

Suppose $(9.5)(a)$ holds.

Since $S$ is continuous at $z$ and $w$ we have

$$
S S x_{2 n+1} \rightarrow S z, S S y_{2 n+1} \rightarrow S w, S\left(F\left(x_{2 n+1}, y_{2 n+1}\right)\right) \rightarrow S z
$$

and

$$
S\left(F\left(y_{2 n+1}, x_{2 n+1}\right)\right) \rightarrow S w .
$$

$\operatorname{Since}(F, S)$ is compatible and by Lemma 1 , we have

$$
\begin{aligned}
\left|d\left(F\left(S x_{2 n+1}, S y_{2 n+1}\right), S z\right)\right| \leq & s\left|d\left(S\left(F\left(x_{2 n+1}, y_{2 n+1}\right)\right), F\left(S x_{2 n+1}, S y_{2 n+1}\right)\right)\right| \\
& +s\left|d\left(S\left(F\left(x_{2 n+1}, y_{2 n+1}\right)\right), S z\right)\right| \rightarrow 0
\end{aligned}
$$


Hence $F\left(S x_{2 n+1}, S y_{2 n+1}\right) \rightarrow S z$.

Similarly,we get $F\left(S y_{2 n+1}, S x_{2 n+1}\right) \rightarrow S w$. Now from $(9.5)(a)(i)$ we have

$$
\begin{aligned}
\alpha\left(\left(S S x_{2 n+1}, S S y_{2 n+1}\right),\left(T x_{2 n+2},\right.\right. & \left.\left.T y_{2 n+2}\right)\right) \\
& =\alpha\left(\left(S z_{2 n}, S w_{2 n}\right),\left(z_{2 n+1}, w_{2 n+1}\right)\right) \geq 1 .
\end{aligned}
$$

Consider

$$
\begin{aligned}
& 1+s^{2} d(S z, z)+s^{2} d(S w, w) \\
& \precsim 1+s^{2}\left[s d\left(S z, S S x_{2 n+1}\right)+s^{2} d\left(S S x_{2 n+1}, T x_{2 n+2}\right)+s^{2} d\left(T x_{2 n+2}, z\right)\right] \\
& \quad \quad+s^{2}\left[s d\left(S w, S S y_{2 n+1}\right)+s^{2} d\left(S S y_{2 n+1}, T y_{2 n+2}\right)+s^{2} d\left(T y_{2 n+2}, z\right)\right] \\
& =\left[1+s^{4} d\left(S S x_{2 n+1}, T x_{2 n+2}\right)+s^{4} d\left(S S y_{2 n+1}, T y_{2 n+2}\right)\right] \\
& \quad+s^{3} d\left(S z, S S x_{2 n+1}\right)+s^{4} d\left(T x_{2 n+2}, z\right)+s^{3} d\left(S w, S S y_{2 n+1}\right)+s^{4} d\left(T y_{2 n+2}, w\right) . \\
& \left|1+s^{2} d(S z, z)+s^{2} d(S w, w)\right| \\
& \leq\left|1+s^{4} d\left(S S x_{2 n+1}, T x_{2 n+2}\right)+s^{4} d\left(S S y_{2 n+1}, T y_{2 n+2}\right)\right|+s^{3}\left|d\left(S z, S S x_{2 n+1}\right)\right| \\
& +s^{4}\left|d\left(T x_{2 n+2}, z\right)\right|+s^{3}\left|d\left(S w, S S y_{2 n+1}\right)\right|+s^{4}\left|d\left(T y_{2 n+2}, w\right)\right| .
\end{aligned}
$$

Letting $n \rightarrow \infty$ and using Lemma 1, we get

$$
\begin{aligned}
& \left|1+s^{2} d(S z, z)+s^{2} d(S w, w)\right| \\
& \quad \leq \lim _{n \rightarrow \infty}\left|1+s^{4} d\left(S S x_{2 n+1}, T x_{2 n+2}\right)+s^{4} d\left(S S y_{2 n+1}, T y_{2 n+2}\right)\right|
\end{aligned}
$$

Now we consider

$$
\begin{aligned}
s^{2}|d(S z, z)|= & s^{4} \frac{1}{s^{2}}|d(S z, z)| \\
\leq & s^{4} \lim _{\mathrm{n} \rightarrow \infty}\left|d\left(F\left(S x_{2 \mathrm{n}+1}, S y_{2 \mathrm{n}+1}\right), G\left(x_{2 \mathrm{n}+2}, y_{2 \mathrm{n}+2}\right)\right)\right| \\
\leq & \lim _{\mathrm{n} \rightarrow \infty} \alpha\left(\left(S S x_{2 \mathrm{n}+1}, S S y_{2 \mathrm{n}+1}\right),\left(T x_{2 \mathrm{n}+2}, T y_{2 \mathrm{n}+2}\right)\right) s^{4} \\
& \left|d\left(F\left(S x_{2 \mathrm{n}+1}, S y_{2 \mathrm{n}+1}\right), G\left(x_{2 \mathrm{n}+2}, y_{2 \mathrm{n}+2}\right)\right)\right| \\
\leq & \lim _{\mathrm{n} \rightarrow \infty}\left[a_{1}\left|d\left(S S x_{2 \mathrm{n}+1}, T x_{2 \mathrm{n}+2}\right)\right|+a_{2}\left|d\left(S S y_{2 \mathrm{n}+1}, T y_{2 \mathrm{n}+2}\right)\right|\right. \\
& +a_{3}\left|d\left(S S x_{2 \mathrm{n}+1}, F\left(S x_{2 \mathrm{n}+1}, S y_{2 \mathrm{n}+1}\right)\right)\right| \\
& +a_{4}\left|d\left(S S y_{2 \mathrm{n}+1}, F\left(S y_{2 \mathrm{n}+1}, S x_{2 \mathrm{n}+1}\right)\right)\right| \\
& +a_{5}\left|d\left(T x_{2 \mathrm{n}+2}, G\left(x_{2 \mathrm{n}+2}, y_{2 \mathrm{n}+2}\right)\right)\right|+a_{6}\left|d\left(T y_{2 \mathrm{n}+2}, G\left(y_{2 \mathrm{n}+2}, x_{2 \mathrm{n}+2}\right)\right)\right| \\
& +a_{7} \frac{\left|d\left(S S x_{2 \mathrm{n}+1}, F\left(S x_{2 \mathrm{n}+1}, S y_{2 \mathrm{n}+1}\right)\right)\right|\left|d\left(T x_{2 \mathrm{n}+2}, G\left(x_{2 \mathrm{n}+2}, y_{2 \mathrm{n}+2}\right)\right)\right|}{\left|1+s^{4} d\left(S S x_{2 \mathrm{n}+1}, T x_{2 \mathrm{n}+2}\right)+s^{4} d\left(S S y_{2 \mathrm{n}+1}, T y_{2 \mathrm{n}+2}\right)\right|} \\
& \left.+a_{8} \frac{\left|d\left(T x_{2 \mathrm{n}+2}, F\left(S x_{2 \mathrm{n}+1}, S y_{2 \mathrm{n}+1}\right)\right)\right|\left|d\left(S S x_{2 \mathrm{n}+1}, G\left(x_{2 \mathrm{n}+2}, y_{2 \mathrm{n}+2}\right)\right)\right|}{\left|1+s^{4} d\left(S S x_{2 \mathrm{n}+1}, T x_{2 \mathrm{n}+2}\right)+s^{4} d\left(S S y_{2 \mathrm{n}+1}, T y_{2 \mathrm{n}+2}\right)\right|}\right] \\
\leq & a_{1} s^{2}|d(S z, z)|+a_{2} s^{2}|d(S w, w)|+a_{8} \frac{s^{2}|d(S z, z)| s^{2}|d(S z, z)|}{\left|1+s^{2} d(S z, z)+s^{2} d(S w, w)\right|},
\end{aligned}
$$


from Lemma 3 and (9)

$<\left(a_{1}+a_{8}\right) s^{2}|d(S z, z)|+a_{2} s^{2}|d(S w, w)|$,

from Remark 1(i).

Thus $|d(z, S z)|<\frac{a_{2}}{1-a_{1}-a_{8}}|d(w, S w)|$.

Similarly using $(9.5)(a)(i)$, we have

$$
|d(w, S w)|<\frac{a_{2}}{1-a_{1}-a_{8}}|d(z, S z)| .
$$

From Remark 1(iii), we have $S z=z$ and $S w=w$.

From $(9.5)(a)(i i)$, we have

$\alpha\left((S z, S w),\left(T x_{2 n+2}, T y_{2 n+2}\right)\right)=\alpha\left((z, w),\left(z_{2 n+1}, w_{2 n+1}\right)\right) \geq 1$.

From Lemma 3:

$$
\begin{aligned}
& \frac{1}{s}|d(F(z, w), z)| \\
& \leq \lim _{n \rightarrow \infty}\left|d\left(F(z, w), G\left(x_{2 n+2}, y_{2 n+2}\right)\right)\right| \\
& \leq \lim _{n \rightarrow \infty} \alpha\left((S z, S w),\left(T x_{2 n+2}, T y_{2 n+2}\right)\right) s^{4}\left|d\left(F(z, w), G\left(x_{2 n+2}, y_{2 n+2}\right)\right)\right| \\
& \leq \lim _{n \rightarrow \infty}\left[\begin{array}{c}
a_{1}\left|d\left(z, z_{2 n+1}\right)\right|+a_{2}\left|d\left(w, w_{2 n+1}\right)\right|+a_{3}|d(z, F(z, w))| \\
a_{4}|d(w, F(w, z))|+a_{5}\left|d\left(z_{2 n+1}, z_{2 n+2}\right)\right|+a_{6}\left|d\left(w_{2 n+1}, w_{2 n+2}\right)\right| \\
+a_{7} \frac{|d(z, F(z, w))|\left|d\left(z_{2 n+1}, z_{2 n+2}\right)\right|}{\left|1+d\left(z, z_{2 n+1}\right)+d\left(w, w_{2 n+1}\right)\right|}+a_{8} \frac{\left|d\left(z_{2 n+1}, F(z, w)\right)\right| d\left(z, z_{2 n+2}\right) \mid}{\left|1+d\left(z, z_{2 n+1}\right)+d\left(w, w_{2 n+1}\right)\right|}
\end{array}\right] \\
& \leq a_{3}|d(z, F(z, w))|+a_{4}|d(w, F(w, z))|, \text { by Lemmas } 1 \text { and 3. }
\end{aligned}
$$

Thus $|d(z, F(z, w))| \leq \frac{a_{4} s}{1-a_{3} s}|d(w, F(w, z))|$.

Similarly using $(9.5)(a)($ ii $)$, we have

$|d(w, F(w, z))| \leq \frac{a_{4} s}{1-a_{3} s}|d(z, F(z, w))|$. From Remark 1(iii), we have $z=F(z, w)$ and $F(w, z)=w$. Thus $S z=z=F(z, w), S w=w=F(w, z)$. Since $z=F(z, w) \in F(X \times X) \subseteq T(X)$ and $w=F(w, z) \in F(X \times X) \subseteq T(X)$, there exist $u$ and $v$ in $X$ such that $z=T u$ and $w=T v$. From $(9.5)(a)(i i i)$,we have $\alpha((S z, S w),(T u, T v))=\alpha((z, w),(z, w)) \geq 1$. Now from $(9.2)$, we have

$$
\begin{aligned}
d(z, G(u, v))= & d(F(z, w), G(u, v)) \\
\precsim & \alpha((S z, S w),(T u, T v)) s^{4} d(F(z, w), G(u, v)) \\
\precsim & a_{1} d(z, z)+a_{2} d(w, w)+a_{3} d(z, z)+a_{4} d(w, w)+a_{5} d(z, G(u, v)) \\
& +a_{6} d(w, G(v, u))+a_{7} \frac{d(z, z) d(z, G(u, v))}{1+s^{4} d(z, z)+s^{4} d(w, w)} \\
& +a_{8} \frac{d(z, z) d(z, G(u, v))}{1+s^{4} d(z, z)+s^{4} d(w, w)} \\
= & a_{5} d(z, G(u, v))+a_{6} d(w, G(v, u)) .
\end{aligned}
$$


Thus $\mid d\left(z, G(u, v)\left|\leq \frac{a_{6}}{1-a_{5}}\right| d(w, G(v, u)) \mid\right.$.

Similarly using $(9.5)(a)($ iii $)$, we have

$$
\mid d\left(w, G(v, u)\left|\leq \frac{a_{6}}{1-a_{5}}\right| d(z, G(u, v)) \mid .\right.
$$

From Remark 1(iii), we have $z=G(u, v)$ and $w=G(v, u)$.

Since the $\operatorname{pair}(G, T)$ is $w$-compatible, we have $T z=G(z, w)$ and $w=$ $G(w, z)$. From $(9.5)(a)(i v)$, we have

$\alpha((S z, S w),(T z, T w))=\alpha((z, w),(T z, T w)) \geq 1$. From $(9.2)$, we have

$$
\begin{aligned}
|d(z, T z)|= & d(F(z, w), G(z, w)) \\
\leq & \alpha((S z, S w),(T z, T w)) s^{4}|d(F(z, w), G(z, w))| \\
\leq & a_{1}|d(z, T z)|+a_{2}|d(w, T w)|+a_{3}|d(z, z)|+a_{4}|d(w, w)| \\
& +a_{5}|d(T z, T z)|+a_{6}|d(T w, T w)|+a_{7} \frac{|d(z, z)||d(T z, G(z, w))|}{\left|1+s^{4} d(z, T z)+s^{4} d(w, T w)\right|} \\
& +a_{8} \frac{|d(z, T z)||d(z, T z)|}{\left|1+s^{4} d(z, T z)+s^{4} d(w, T w)\right|} \\
\leq & a_{1}|d(z, T z)|+a_{2}|d(w, T w)|+a_{8} s^{4} \frac{|d(z, T z)||d(z, T z)|}{\left|1+s^{4} d(z, T z)+s^{4} d(w, T w)\right|} \\
\leq & a_{1}|d(z, T z)|+a_{2}|d(w, T w)|+a_{8}|d(z, T z)|, \operatorname{fromRemark} 1(i) .
\end{aligned}
$$

Thus $|d(z, T z)|<\frac{a_{2}}{1-a_{1}-a_{8}}|d(w, T w)|$.

Similarly using $(9.5)(a)(i v)$, we have

$$
|d(w, T w)|<\frac{a_{2}}{1-a_{1}-a_{8}}|d(z, T z)| .
$$

Thus from Remark 1(iii), we have $T z=z$ and $T w=w$. Thus $(z, w)$ is a common coupled fixed point of $F, G, S$ and $T$.

Uniqueness of common coupled fixed point of $F, G, S$ and $T$ follows easily from (9.6) and (9.2).

Now we give an example to illustrate Theorem 9.

Example 1. Let $X=[0,2]$ and $d(x, y)=i|x-y|^{2}, \forall x, y \in X$.

Define $F, G: X \times X \rightarrow X$ by $F(x, y)=\frac{x^{2}+y^{2}}{24}$ and $G(x, y)=\frac{x^{2}+y^{2}}{36}$ and $S, T: X \rightarrow X$ by $S x=\frac{x^{2}}{2}$ and $T x=\frac{x^{2}}{3}$.

Define $\alpha: X^{2} \times X^{2} \rightarrow \mathcal{R}^{+}$by

$$
\alpha((x, y),(u, v))= \begin{cases}1, & \text { if } x, y, u, v \in[0, \sqrt{3}] \\ 0, & \text { otherwise }\end{cases}
$$


Clearly $F(X \times X) \subseteq T(X)$ and $G(X \times X) \subseteq S(X)$. To verify (9.2) we consider the following two cases.

Case (a) Suppose $x, y, u, v \in[0, \sqrt{3}]$.

Then $\alpha((S x, S y),(T u, T v))=\alpha\left(\left(\frac{x^{2}}{2}, \frac{y^{2}}{2}\right),\left(\frac{u^{2}}{3}, \frac{v^{2}}{3}\right)\right)=1$,

$$
\begin{aligned}
\alpha((S x, S y),(T u, T v)) & \left.s^{4} d(F(x, y), G(u, v))\right) \\
& =16 i\left|\frac{x^{2}+y^{2}}{24}-\frac{u^{2}+v^{2}}{36}\right|^{2} \\
& =\frac{16}{144} i\left|\frac{3 x^{2}-2 u^{2}+3 y^{2}-2 v^{2}}{6}\right|^{2} \\
& \leq \frac{2}{9} i\left[\left|\frac{3 x^{2}-2 u^{2}}{6}\right|^{2}+\left|\frac{3 y^{2}-2 v^{2}}{6}\right|^{2}\right] \\
& =\frac{2}{9}[d(S x, T u)+d(S y, T v)] .
\end{aligned}
$$

Here $a_{1}=a_{2}=\frac{2}{9}, a_{3}=a_{4}=0$. Clearly $\sum_{i=1}^{4} a_{i}<\frac{1}{s}$.

Case (b): Atleast one of $x, y, u, v \notin[0, \sqrt{3}]$.

Then $\alpha((S x, S y),(T u, T v))=\alpha\left(\left(\frac{x^{2}}{2}, \frac{y^{2}}{2}\right),\left(\frac{u^{2}}{3}, \frac{v^{2}}{3}\right)\right)$.

Sub case: If $\frac{x^{2}}{2}, \frac{y^{2}}{2}, \frac{u^{2}}{3}, \frac{v^{2}}{3} \in(1, \sqrt{3}]$ then

$$
\alpha((S x, S y),(T u, T v))=1 .
$$

The inequality (9.2) holds as in case (a).

Sub case: If atleast one of If $\frac{x^{2}}{2}, \frac{y^{2}}{2}, \frac{u^{2}}{3}, \frac{v^{2}}{3} \in(\sqrt{3}, 2]$ then

$$
\alpha((S x, S y),(T u, T v))=0 .
$$

Hence (9.2) holds.

By definition of $\alpha$, the condition (9.3) with $x_{1}=0=y_{1}$ and (9.4) are satisfied clearly.

To verify the compatibility of the pair $(F, S)$, let us consider the sequences $\left\{x_{n}\right\},\left\{y_{n}\right\}$ in $X$ such that $F\left(x_{n}, y_{n}\right) \rightarrow t, S x_{n} \rightarrow t, F\left(y_{n}, x_{n}\right) \rightarrow t^{\prime}$ and $S x_{n} \rightarrow t^{\prime}$ for some $t, t^{\prime} \in X, F\left(x_{n}, y_{n}\right) \rightarrow t \Rightarrow \frac{x_{\mathrm{n}}^{2}+y_{\mathrm{n}}^{2}}{24} \rightarrow t$ and $F\left(y_{n}, x_{n}\right) \rightarrow t^{\prime} \Rightarrow \frac{y_{\mathrm{n}}^{2}+x_{\mathrm{n}}^{2}}{24} \rightarrow$ $t^{\prime}$. Hence $t=t^{\prime}, S x_{n} \rightarrow t \Rightarrow \frac{x_{n}^{2}}{2} \rightarrow t$ and $S y_{n} \rightarrow t^{\prime} \Rightarrow \frac{y_{n}^{2}}{2} \rightarrow t^{\prime}=t$. 
Now $\frac{x_{\mathrm{n}}^{2}+y_{\mathrm{n}}^{2}}{24} \rightarrow \frac{4 t^{2}+4 t^{2}}{24}=\frac{t^{2}}{3}$. Hence $\frac{t^{2}}{3}=t \Rightarrow t=0$. Thus $x_{n}^{2}, y_{n}^{2} \rightarrow 0$.

$$
\left|d\left(S\left(F\left(x_{n}, y_{n}\right)\right), F\left(S x_{n}, S y_{n}\right)\right)\right|=\left|\frac{1}{2}\left(\frac{x_{n}^{2}+y_{n}^{2}}{24}\right)^{2}-\frac{1}{24}\left(\frac{x_{n}^{4}}{4}+\frac{y_{n}^{4}}{4}\right)\right|^{2} \rightarrow 0
$$

as $n \rightarrow \infty$.

Similarly $\left|d\left(S\left(F\left(y_{n}, x_{n}\right)\right), F\left(S y_{n}, S x_{n}\right)\right)\right| \rightarrow 0$. Thus the pair $(F, S)$ is compatible. Also it is clear that the pair $(G, T)$ is $w$-compatible.

By definition of $\alpha$, one can easily verify the conditions (9.5) and (9.6).

Thus all the conditions of Theorem 9 are satisfied and $(0,0)$ is the unique common fixed point of $F, G, S$ and $T$.

If $\alpha((x, y),(u, v))=1$ for all $x, y, u, v \in X$ and $s=1$ in Theorem 9 , we have the following corollary.

Corollary 10. Let $(X, d)$ be a complete complex valued metric space. Let $F, G: X \times X \rightarrow X$ and $S, T: X \rightarrow X$ be mappings satisfying the following :

$$
F(X \times X) \subseteq T(X), G(X \times X) \subseteq S(X)
$$

$$
\begin{aligned}
& d(F(x, y), G(u, v)) \\
& \precsim a_{1} d(S x, T u)+a_{2} d(S y, T v)+a_{3} d(S x, F(x, y)) \\
& \quad+a_{4} d(S y, F(y, x))+a_{5} d(T u, G(u, v)) \\
& +a_{6} d(T v, G(v, u))+a_{7} \frac{d(S x, F(x, y)) d(T u, G(u, v))}{1+d(S x, T u)+d(S y, T v)} \\
& \quad+a_{8} \frac{d(T u, F(x, y)) d(S x, G(u, v))}{1+d(S x, T u)+d(S y, T v)}
\end{aligned}
$$

for all $x, y, u, v \in X$, where $a_{i}, i=1,2, \ldots . ., 8$ are non-negative real numbers such that $\sum_{i=1}^{8} a_{i}<1$,

$(10.3)(a)$ the pair $(F, S)$ is compatible, $S$ is continuous and the pair $(G, T)$ is $w$-compatible

(or)

(b) the pair $(G, T)$ is compatible, $T$ is continuous and the pair $(F, S)$ is $w$-compatible

Then $F, G, S$ and $T$ have a unique common coupled fixed point. 


\section{References}

[1] A. Azam, B. Fisher, M. Khan, Common fixed point theorems in complex valued metric spaces, Numer. Funct. Anal. Optim., 32, No. 3 (2011), 243-253, doi: 10.1080/01630563.

[2] B. Samet, C. Vetro, P. Vetro, Fixed point theorems for $\alpha-\psi$-contractive type mappings, Nonlinear Analysis, 75 (2012), 2154-2165, doi: 10.1016/j.na.2011.10.014.

[3] C. Klin-eam, C. Suanoom, Some common fixed point theorems for generralized contractive type mappings on complex valued metric spaces, Abstr. Appl. Anal. (2013), 6 pages, Article ID 604215, doi: 10.1155/2013/604215.

[4] F. Rouzkard, M. Imdad, Some common fixed point theorems on complex valued metric spaces, Comp. Math. Appls., 64 (2012), 1866-1874, doi: 10.1016/j.camwa.2012.02.063.

[5] G. Jungck, Compatible mappings and common fixed points, Int. J. Math. Math. Sci., 9, 4 (1986), 771-779, doi: 10.1155/S0161171286000935.

[6] H.K. Nashine, M. Imdad, M. Hasan, Common fixed point theorems under rational contractions in complex valued metric spaces, J. Nonlinear Sci. Appl., 7 (2014), 42-50.

[7] J. Kumar, S. Vashistha, Coupled fixed point theorem for generalized contraction in complex valued metric spaces, International Journal of Computer Applications, 83, No. 7 (2013), 36-40, doi: 10.5120/14463-2745.

[8] K. Sitthikul, S. Saejung, Some fixed points in complex valued metric spaces, Fixed point theory Appl. (2012), 11 pages, Article ID 189, doi: 10.1186/1687-1812-2012-189.

[9] K.P.R. Rao, P. Ranga Swamy, J. Rajendra Prasad, A common fixed point theorem in complex valued $b$-metric spaces, Bulletin of Mathematics and Statistics Research, 1, No. 1 (2013), 1-8.

[10] M. Abbas, M. Ali Khan, S. Radenovic, Common coupled fixed point theorems in cone metric spaces for w-compatible mappings, Appl. Math. Comput, 217 (2010), 195-202, doi: 10.1016/j.amc.2010.05.042.

[11] M. Abbas, B. Fisher, T. Nazir, Well-posedness and peroidic point property of mappings satisfying a rational inequality in an ordrerd complex valued-metric spaces, Sci. Stud. Res., Ser. Math. Inform., 22, No. 1 (2012), 5-24.

[12] M. Abbas, M. Arshad, A. Azam, Fixed points of asympotically regular mappings in complex valued metric spaces, Georgian Math. J., 20 (2013), 213-221, doi: 10.1515/gmj2013-0013.

[13] M. A. Kutbi, A. Azam, J. Ahmad, C. Di Bari, Some common coupled fixed point results for generalized contraction in complex-valued metric spaces, J. Appl. Math. (2013), 10 pages, ArticleID 352927, doi: 10.1155/2013/352927.

[14] M. Kumar, P. Kumar, S. Kumar, Common fixed point theorems in complex valued metric spaces, J. Ana. Num. Theor., 2, No. 2 (2014), 103-109, doi: 10.12785/jant/020213.

[15] M. Musaleen, S.A. Mohiuddine, R.P. Agarwal, Coupled fixed point theorems for $(\alpha-\psi)$ contractive type mappings in partially ordered metric spaces, Fixed Point Theory and Applications (2012), 2012:228, 11 pages, doi: 10.1186/1687-1812-2012-228.

[16] N. Singh, D. Singh, A. Badal, V. Joshi, Fixed point theorems in complex valued metric spaces, Journal of the Egyptian Mathematical Society (2015), 1-8, doi: 10.1016/j.joems.2015.04.005. 
[17] N. Malhotra, B. Bansal, Some common coupled fixed point theorems for generalized contraction in b-metric spaces, J. Nonlinear Sci. Appl., 8 (2015), 8-16.

[18] P. Kaushik, S. Kumar, P. Kumam, Coupled coincidence point theorems for $\alpha-\psi$ contractive type mappings in partially ordered metric spaces, Fixed Point Theory and Applications (2013), 325, 10 pages, doi: 10.1186/1687-1812-2013-325.

[19] P. Shahi, J. Kumar, S.S. Bhatia, Coincidence and common fixed point results for generalized $\alpha-\psi$-contractive type mappings with applications, Bull. Belg. Math. Soc. Simon Stevin, 22, No. 2 (2015), 299-318.

[20] R. K. Verma, H. K. Pathak, Common fixed point theorems for a pair of mappings in complex valued metric spaces, Journal of Mathematics and Computer Science, 6 (2013), $18-26$.

[21] S. Chandok, D. Kumar, Some common fixed point results for rational type contraction mappings in complex valued metric spaces, Journal of Operators (2013), 6 pages, Article ID 813707, doi: 10.1155/2013/813707.

[22] S.M. Kang, Coupled fixed point theorems in complex valued metric spaces, Int. Journal of Math. Analysis, 7, No. 46 (2013), 2269-2277, doi: 10.12988/ijma.2013.37181.

[23] T.G. Bhaskar, V. Lakshmikantham, Fixed point theorems in partially ordered metric spaces and applications, Nonlinear Analysis, 65, No. 7 (2006), 1379-1393, doi: 10.1016/j.na.2005.10.017.

[24] T. Mitra, A common coupled fixed point result in complex valued metric space for two mappings, International Journal of Current Research, 7, No. 8 (2015), 19555-19559.

[25] T. Mitra, A common coupled fixed point theorem in complex valued metric space for two mappings satisfying a rational inequality, International Journal of Scientific Research Engineering and Technology, 4, No. 8 (2015), 831-835.

[26] T. Senthil Kumar, R. Jahir Hussain, Common coupled fixed point theorem for contractive type mappings in closed ball of complex valued metric spaces, Adv. Inequal. Appl. (2014), $1-7,34$.

[27] V. Lakshmikantham, Lj. Ciric, Coupled fixed point theorems for nonlinear contractions in partially ordered metric spaces, Nonlinear Analysis:Theory, Method. Appl., 70, No. 12 (2009), 4341-4349, doi: 10.1016/j.na.2008.09.020.

[28] W. Sintunavarat, P. Kumam, Generalized common fixed point theorems in complex valued metric spaces and applications, J. Inequal. appl., (2012), 11 pages, Article ID 84, doi: 10.1186/1029-242X-2012-84. 